TAIWANESE JOURNAL OF MATHEMATICS

Vol. 17, No. 1, pp. 161-178, February 2013

DOI: $10.11650 /$ tjm.17.2013.2127

This paper is available online at http://journal.taiwanmathsoc.org.tw

\title{
PRODUCTS OF RADIAL DERIVATIVE AND MULTIPLICATION OPERATORS FROM $F(p, q, s)$ TO WEIGHTED-TYPE SPACES ON THE UNIT BALL
}

Jie Zhou and Yongmin Liu*

\begin{abstract}
In this paper, we obtain the complete characterizations of the boundedness and compactness of the products of the radial derivative and the multiplication operator $\mathcal{R} M_{u}$ from $F(p, q, s)$ to weighted-type spaces on the unit ball.
\end{abstract}

\section{INTRODUCTION}

Let $z=\left(z_{1}, \cdots, z_{n}\right)$ and $w=\left(w_{1}, \cdots, w_{n}\right)$ be points in the complex vector space $\mathbb{C}^{n}$ and $z \bar{w}:=\langle z, w\rangle=z_{1} \overline{w_{1}}+z_{2} \overline{w_{2}}+\cdots+z_{n} \overline{w_{n}}$. We also write

$$
|z|=\sqrt{\langle z, z\rangle}=\sqrt{\sum_{j=1}^{n}\left|z_{j}\right|^{2}} .
$$

Let $\mathbb{B}=\left\{z \in \mathbb{C}^{n}:|z|<1\right\}$ be the open unit ball in $\mathbb{C}^{n}, S=\partial \mathbb{B}$ its boundary, and $H(\mathbb{B})$ denote the class of all holomorphic functions on $\mathbb{B}$. For $f \in H(\mathbb{B})$ with the Taylor expansion $f(z)=\sum_{|\beta| \geq 0} a_{\beta} z^{\beta}$, let $\mathcal{R} f(z)=\sum_{|\beta| \geq 0}|\beta| a_{\beta} z^{\beta}$ be the radial derivative of $f$ at $z$, where $\beta=\left(\beta_{1}, \beta_{2}, \cdots, \beta_{n}\right)$ is a multi-index, $|\beta|=\beta_{1}+\beta_{2}+\cdots+\beta_{n}$ and $z^{\beta}=z_{1}^{\beta_{1}} z_{2}^{\beta_{2}} \cdots z_{n}^{\beta_{n}}$. It is easy to see that (see, e.g., $[20,48]$ )

$$
\mathcal{R} f(z)=\sum_{j=1}^{n} z_{j} \frac{\partial f}{\partial z_{j}}(z)
$$

The iterated radial derivative operator $\mathcal{R}^{m} f$ is defined inductively by $([4,5,27])$ :

Received June 12, 2012, accepted June 22, 2012.

Communicated by Alexander Vasiliev.

2010 Mathematics Subject Classification: 47B38, 47G10, 32A10, 32A18.

Key words and phrases: Unit ball, General space, Weighted-type space, Radial derivative operator, Multiplication operator.

Supported by the Natural Science Foundation of China (11171285) and the Priority Academic Program Development of Jiangsu Higher Education Institutions.

*Corresponding author. 


$$
\mathcal{R}^{m} f=\mathcal{R}\left(\mathcal{R}^{m-1} f\right), m \in \mathbb{N}-\{1\} .
$$

A positive continuous function $\mu$ on $[0,1)$ is called normal, if there is a $\delta \in[0,1)$ and $a$ and $b, 0<a<b$ such that (see, e.g., $[13,21]$ )

$$
\begin{aligned}
& \frac{\mu(r)}{(1-r)^{a}} \text { is decreasing on }[\delta, 1) \text { and } \lim _{r \rightarrow 1} \frac{\mu(r)}{(1-r)^{a}}=0, \\
& \frac{\mu(r)}{(1-r)^{b}} \text { is increasing on }[\delta, 1) \text { and } \lim _{r \rightarrow 1} \frac{\mu(r)}{(1-r)^{b}}=\infty .
\end{aligned}
$$

If we say that a function $\mu: \mathbb{B} \rightarrow[0, \infty)$ is normal, we also assume that it is radial, that is, $\mu(z)=\mu(|z|), z \in \mathbb{B}$. The weighted-type space $H_{\mu}^{\infty}(\mathbb{B})=H_{\mu}^{\infty}$ consists of all $f \in H(\mathbb{B})$ such that

$$
\|f\|_{H_{\mu}^{\infty}}:=\sup _{z \in \mathbb{B}} \mu(z)|f(z)|<\infty
$$

where $\mu$ is a weight (see, e.g., [2] as well as [1] for a related class of spaces).

The little weighted-type space $H_{\mu, 0}^{\infty}(\mathbb{B})=H_{\mu, 0}^{\infty}$ is a subspace of $H_{\mu}^{\infty}$ consisting of all $f \in H(\mathbb{B})$ such that

$$
\lim _{|z| \rightarrow 1} \mu(|z|)|f(z)|=0 .
$$

The Bloch-type space $\mathcal{B}^{\alpha}(\alpha>0)$ consists of all $f \in H(\mathbb{B})$ such that

$$
\|f\|_{\mathcal{B}^{\alpha}}=|f(0)|+\sup _{z \in \mathbb{B}}\left(1-|z|^{2}\right)^{\alpha}|\mathcal{R} f(z)|<\infty .
$$

Let $0<p, s<\infty,-n-1<q<\infty$. A function $f \in H(\mathbb{B})$ is said to belong to $F(p, q, s)=F(p, q, s)(\mathbb{B})$ (see, e.g., $[6,43,46])$ if

$$
\|f\|_{F(p, q, s)}^{p}=|f(0)|^{p}+\sup _{a \in \mathbb{B}} \int_{\mathbb{B}}|\mathcal{R} f(z)|^{p}\left(1-|z|^{2}\right)^{q} g^{s}(z, a) d V(z)<\infty,
$$

where $g(z, a)=\log \left|\varphi_{a}(z)\right|^{-1}$ is the Green's function for $\mathbb{B}$ with logarithmic singularity at $a, d V$ is the normalized Lebesgue measure on $\mathbb{C}^{n}$. We call $F(p, q, s)$ general function space because we can get many function spaces, such as BMOA space, $Q_{p}$ space, Bergman space, Hardy space, Bloch space, if we take special parameters of $p, q, s$. If $q+s \leqslant-1$, then $F(p, q, s)$ is the space of constant functions.

The weighted iterated radial-derivative composition operator is defined by S. Stevic in [27] and [30] as follows:

$$
\mathcal{R}_{u, \varphi}^{m} f(z)=\left(M_{u} C_{\varphi} \mathcal{R}^{m}\right) f(z)=u(z) \mathcal{R}^{m} f(\varphi(z)), z \in \mathbb{B} .
$$

Some characterization for the boundedness and compactness of the operator $\mathcal{R}_{u, \varphi}^{m}$ between various spaces of holomorphic function on the unit ball can be found in [27, 30]. Some related operators between $F(p, q, s)$ spaces and various spaces on the unit ball, are 
treated, for example (see, e.g., $[10,13,18,24,31,33,35,38,39,40,41,45,47,50]$ ), when $m=1$ and $\varphi(z)=z$, we can get the operator $M_{u} \mathcal{R}$. For related one-dimensional operators, see, for example [7, 8, 9, 11, 12, 14, 15, 16, 25, 26, 28, 29, 32, 37, 42, 49], as well as the related references therein. The boundedness and compactness of the operator $M_{u} \mathcal{R}$ from mixed norm spaces $H(p, q, \phi)$ to Zygmund-type spaces on the unit ball have been studied, for example, in [17]. The boundedness and compactness of the operator $M_{u} \mathcal{R}$ from mixed norm spaces $H(p, q, \phi)$ to the $n$th weighted-type space on the unit ball have been studied, for example, in [34]. Inspired by these results, we can define the operator $\mathcal{R} M_{u}$ as follows:

$$
\begin{aligned}
\mathcal{R} M_{u} f(z) & =\mathcal{R}(u(z) f(z)) \\
& =u(z) \sum_{j=1}^{n} z_{j} \frac{\partial f}{\partial z_{j}}(z)+\sum_{j=1}^{n} z_{j} \frac{\partial u}{\partial z_{j}}(z) f(z) \\
& =u(z) \mathcal{R} f(z)+\mathcal{R} u(z) f(z) \\
& =M_{u} \mathcal{R} f(z)+\mathcal{R} u(z) f(z) .
\end{aligned}
$$

The purpose of this paper is to study the boundedness and compactness of the operator $\mathcal{R} M_{u}$ from $F(p, q, s)$ spaces to weighted-type spaces on the unit ball.

\section{Auxiliary Results}

Here we state several auxiliary results most of which will be used in the proofs of the main results. The following lemma can be found in [43].

Lemma 1. Assume that $0<p, s<\infty,-n-1<q<\infty, q+s>-1$ and $f \in$ $F(p, q, s)$, then $f \in \mathcal{B}^{\frac{n+1+q}{p}}$ and $\|f\|_{\mathcal{B}^{\frac{n+1+q}{p}}} \leq C\|f\|_{F(p, q, s)}$.

The next folklore lemma can be found in [22].

Lemma 2. Assume that $f \in \mathcal{B}^{\alpha}, \alpha>0$, then for any $z \in \mathbb{B}$

$$
|f(z)| \leq C\left\{\begin{array}{l}
|f(0)|+\|f\|_{\mathcal{B}^{\alpha}}, 0<\alpha<1 . \\
|f(0)|+\|f\|_{\mathcal{B}^{\alpha}} \cdot \log \frac{2}{1-|z|^{2}}, \quad \alpha=1 . \\
|f(0)|+\frac{\|f\|_{\mathcal{B}^{\alpha}}}{\left(1-|z|^{2}\right)^{\alpha-1}}, \quad \alpha>1 .
\end{array}\right.
$$

To investigate the compactness of the operator $\mathcal{R} M_{u}$, we also need the next lemma. For the case $\mu(z)=1-|z|^{2}$, the lemma was proved in [19]. For the general case the proof is similar, we omit the details.

Lemma 3. Let $\mu$ be a normal function. A closed set $K$ in $H_{\mu, 0}^{\infty}(\mathbb{B})$ is compact if and only if it is bounded and satisfies

$$
\lim _{|z| \rightarrow 1} \sup _{f \in K} \mu(|z|)|f(z)|=0 .
$$


The next Schwartz-type lemma ([41]) is proved in a standard way (see, e.g. [23, Lemma 3]).

Lemma 4. Assume that $0<p, s<\infty,-n-1<q<\infty, \mu$ is a normal function on $[0,1)$, then $\mathcal{R} M_{u}: F(p, q, s) \rightarrow H_{\mu}^{\infty}\left(H_{\mu, 0}^{\infty}\right)$ is compact if and only if $\mathcal{R} M_{u}: F(p, q, s) \rightarrow H_{\mu}^{\infty}\left(H_{\mu, 0}^{\infty}\right)$ is bounded, and for any bounded sequence $\left\{f_{k}\right\}_{k \in \mathbb{N}}$ in $F(p, q, s)$ which converges to zero uniformly on the compact subsets of $\mathbb{B}$ as $k \rightarrow \infty$, we have

$$
\lim _{k \rightarrow \infty}\left\|\mathcal{R} M_{u} f_{k}\right\|_{H_{\mu}^{\infty}}=0
$$

Lemma 5. (see $[10,18])$. Let $p=n+1+q, \forall w \in \mathbb{B},\left|g_{w}(z)\right| \leq \frac{C}{\mid 1-\langle z, w\rangle}$, then

$$
\int_{\mathbb{B}}\left|g_{w}(z)\right|^{p}\left(1-|z|^{2}\right)^{q} g^{s}(z, a) d V(z) \leq C .
$$

3. The Boundedness And Compactness of $\mathcal{R} M_{u}: F(p, q, s) \rightarrow H_{\mu}^{\infty}\left(H_{\mu, 0}^{\infty}\right)$

In this section we characterize the boundedness and compactness of $\mathcal{R} M_{u}: F(p, q, s)$ $\rightarrow H_{\mu}^{\infty}\left(H_{\mu, 0}^{\infty}\right)$.

Case 3.1. $p<q+n+1$

Theorem 1. Assume that $0<p, s<\infty,-n-1<q<\infty, q+s>-1, p<q+n+1$ and $\mu$ is a normal weight. Then $\mathcal{R} M_{u}: F(p, q, s) \rightarrow H_{\mu}^{\infty}$ is bounded if and only if

$$
\sup _{z \in \mathbb{B}} \frac{\mu(|z|)|\mathcal{R} u(z)|}{\left(1-|z|^{2}\right)^{\frac{n+1+q}{p}}-1}<\infty,
$$

and

$$
\sup _{z \in \mathbb{B}} \frac{\mu(|z|)|u(z)|}{\left(1-|z|^{2}\right)^{\frac{n+1+q}{p}}}<\infty
$$

Proof. First let us assume that conditions (1) and (2) hold. For any $f \in F(p, q, s)$, by Lemma 1 and Lemma 2, we have

$$
\begin{aligned}
& \mu(|z|)\left|\mathcal{R} M_{u} f(z)\right| \\
= & \mu(|z|)|\mathcal{R} u(z) f(z)+u(z) \mathcal{R} f(z)| \\
\leq & C\|f\|_{\mathcal{B}^{\frac{n+1+q}{p}}}\left(\frac{\mu(|z|)|\mathcal{R} u(z)|}{\left(1-|z|^{2}\right)^{\frac{n+1+q}{p}-1}}+\frac{\mu(|z|)|u(z)|}{\left(1-|z|^{2}\right)^{\frac{n+1+q}{p}}}\right) \\
\leq & C\|f\|_{F(p, q, s)}\left(\frac{\mu(|z|)|\mathcal{R} u(z)|}{\left(1-|z|^{2}\right)^{\frac{n+1+q}{p}}-1}+\frac{\mu(|z|)|u(z)|}{\left(1-|z|^{2}\right)^{\frac{n+1+q}{p}}}\right) .
\end{aligned}
$$


From this, conditions (1) and (2), we can get the operator $\mathcal{R} M_{u}: F(p, q, s) \rightarrow H_{\mu}^{\infty}$ is bounded. Conversely, assume that the operator $\mathcal{R} M_{u}: F(p, q, s) \rightarrow H_{\mu}^{\infty}$ is bounded. Then for any $f \in F(p, q, s)$, there is a positive constant $C$ independent of $f$ such that $\left\|\mathcal{R} M_{u} f\right\|_{H_{\mu}^{\infty}} \leq C\|f\|_{F(p, q, s)}$. Taking the test function $f(z) \equiv 1 \in F(p, q, s)$, we see that

$$
\sup _{z \in \mathbb{B}} \mu(|z|)|\mathcal{R} u(z)|<\infty
$$

For $\omega \in \mathbb{B}$, set

$$
f_{\omega}(z)=\frac{\left(1-|\omega|^{2}\right)^{1+\frac{n+1+q}{p}}}{(1-\langle z, \bar{\omega}\rangle)^{\frac{2(n+1+q)}{p}}}-\frac{\left(1-|\omega|^{2}\right)}{(1-\langle z, \bar{\omega}\rangle)^{\frac{n+1+q}{p}}}, z \in \mathbb{B},
$$

then

$$
\mathcal{R} f_{\omega}(z)=2 A \frac{\left(1-|\omega|^{2}\right)^{1+\frac{n+1+q}{p}} \cdot(z \bar{\omega})}{(1-\langle z, \bar{\omega}\rangle)^{\frac{2(n+1+q)}{p}+1}}-A \frac{\left(1-|\omega|^{2}\right) \cdot(z \bar{\omega})}{(1-\langle z, \bar{\omega}\rangle)^{\frac{n+1+q}{p}+1}}, z \in \mathbb{B}
$$

where $A=\frac{n+1+q}{p}$. It is easy to see that $f_{\omega} \in F(p, q, s)$ for each $\omega \in \mathbb{B}$ and $\sup _{\omega \in \mathbb{B}}\left\|f_{\omega}\right\|_{F(p, q, s)} \leq C$ by using the same methods as in [43], and

$$
f_{\omega}(\omega)=0, \mathcal{R} f_{\omega}(\omega)=A \frac{|\omega|^{2}}{\left(1-|\omega|^{2}\right)^{\frac{n+1+q}{p}}}
$$

Thus for any $w \in \mathbb{B}$, we get

$$
\begin{aligned}
& |A| \frac{\mu(|\omega|)|u(\omega)||\omega|^{2}}{\left(1-|\omega|^{2}\right)^{\frac{n+1+q}{p}}}=\mu(|\omega|)\left|u(\omega) \mathcal{R} f_{\omega}(\omega)\right| \\
\leq & \mu(|\omega|)\left|\mathcal{R} u(\omega) f_{\omega}(\omega)+u(\omega) \mathcal{R} f_{\omega}(\omega)\right| \\
\leq & \left\|\mathcal{R} M_{u}\left(f_{\omega}\right)\right\|_{H_{\mu}^{\infty}} \leq C\left\|\mathcal{R} M_{u}\right\|_{F(p, q, s) \rightarrow H_{\mu}^{\infty}} .
\end{aligned}
$$

Let $r \in(0,1)$, we have

$$
\begin{gathered}
\sup _{r<|\omega|<1} \frac{\mu(|\omega|)|u(\omega)|}{\left(1-|\omega|^{2}\right)^{\frac{n+1+q}{p}}} \\
<\frac{1}{r^{2}} \sup _{r<|\omega|<1} \frac{\mu(|\omega|)|u(\omega)| \cdot|\omega|^{2}}{\left(1-|\omega|^{2}\right)^{\frac{n+1+q}{p}}} \\
\leq C\left\|\mathcal{R} M_{u} f_{\omega}\right\|_{H_{\mu}^{\infty}} \leq C\left\|\mathcal{R} M_{u}\right\|_{F(p, q, s) \rightarrow H_{\mu}^{\infty}} .
\end{gathered}
$$


Using the fact

$$
\begin{aligned}
& \sup _{|\omega| \leq r} \frac{\mu(|\omega|)|u(\omega)|}{\left(1-|\omega|^{2}\right)^{\frac{n+1+q}{p}}} \\
\leq & \frac{1}{\left(1-r^{2}\right)^{\frac{n+1+q}{p}}} \sup _{|\omega| \leq r} \mu(|\omega|)|u(\omega)|<C .
\end{aligned}
$$

Combining (9) and (10), we get (2). To prove (1), let $\omega \in \mathbb{B}$ and set

$$
g_{\omega}(z)=\frac{1-|\omega|^{2}}{(1-\langle z, \bar{\omega}\rangle)^{\frac{n+1+q}{p}}}
$$

Then

$$
\mathcal{R} g_{\omega}(z)=A \frac{\left(1-|\omega|^{2}\right)(z \bar{\omega})}{(1-\langle z, \bar{\omega}\rangle)^{\frac{n+1+q}{p}+1}} .
$$

It is well known $g_{\omega} \in F(p, q, s)$ and $\sup _{\omega \in \mathbb{B}}\left\|g_{\omega}\right\|_{F(p, q, s)} \leq C$ (see, e.g., [43]), and we have

$$
g_{\omega}(\omega)=\frac{1}{\left(1-|\omega|^{2}\right)^{\frac{n+1+q}{p}-1}}, \mathcal{R} g_{\omega}(\omega)=A \frac{|\omega|^{2}}{\left(1-|\omega|^{2}\right)^{\frac{n+1+q}{p}}}
$$

For any $\omega \in \mathbb{B}$, by using (2), (12) and the triangle inequality we get

$$
\begin{aligned}
& \frac{\mu(|\omega|)|\mathcal{R} u(\omega)|}{\left(1-|\omega|^{2}\right)^{\frac{n+1+q}{p}-1}} \\
\leq & \mu(|\omega|)\left|\mathcal{R} u(\omega) g_{\omega}(\omega)+u(\omega) \mathcal{R} g_{\omega}(\omega)\right|+|A| \frac{\mu(|\omega|)|u(\omega)||\omega|^{2}}{\left(1-|\omega|^{2}\right)^{\frac{n+1+q}{p}}} \\
\leq & \left\|\mathcal{R} M_{u} g_{\omega}\right\|_{H_{\mu}^{\infty}}+C \leq C\left\|\mathcal{R} M_{u}\right\|_{F(p, q, s) \rightarrow H_{\mu}^{\infty}}+C .
\end{aligned}
$$

From this, we can get (1), finishing the proof of the theorem.

Theorem 2. Assume that $0<p, s<\infty,-n-1<q<\infty, q+s>-1, p<q+n+1$ and $\mu$ is a normal weight, then the following statements are equivalent:

(A) $\mathcal{R} M_{u}: F(p, q, s) \rightarrow H_{\mu}^{\infty}$ is compact;

(B) $\mathcal{R} M_{u}: F(p, q, s) \rightarrow H_{\mu, 0}^{\infty}$ is compact;

(C)

$$
\lim _{|z| \rightarrow 1} \frac{\mu(|z|)|\mathcal{R} u(z)|}{\left(1-|z|^{2}\right)^{\frac{n+1+q}{p}-1}}=0
$$


and

$$
\lim _{|z| \rightarrow 1} \frac{\mu(|z|)|u(z)|}{\left(1-|z|^{2}\right)^{\frac{n+1+q}{p}}}=0 .
$$

Proof. $(B) \Rightarrow(A)$. This implication is obvious.

$(A) \Rightarrow(C)$. Suppose that the operator $\mathcal{R} M_{u}: F(p, q, s) \rightarrow H_{\mu}^{\infty}$ is compact, then $\mathcal{R} M_{u}: F(p, q, s) \rightarrow H_{\mu}^{\infty}$ is bounded. Let $\left\{z_{k}\right\}$ be a sequence in $\mathbb{B}$ such that $\left|z_{k}\right| \rightarrow 1$ as $k \rightarrow \infty$. Set $f_{k}(z)=f_{z_{k}}(z)$, and we can have

$$
f_{k}(z)=\frac{\left(1-\left|z_{k}\right|^{2}\right)^{\frac{n+1+q}{p}+1}}{\left(1-\left\langle z, z_{k}\right\rangle\right)^{\frac{2(n+1+q)}{p}}}-\frac{\left(1-\left|z_{k}\right|^{2}\right)}{\left(1-\left\langle z, z_{k}\right\rangle\right)^{\frac{n+1+q}{p}}}, k \in \mathbb{N} .
$$

It is easy to see $f_{k} \in F(p, q, s), \sup _{k \in \mathbb{N}}\left\|f_{k}\right\|_{F(p, q, s)} \leq C$ and $f_{k}$ converges to zero uniformly on the compact subsets of $\mathbb{B}$, using Lemma 4 , we get $\lim _{k \rightarrow \infty}\left\|\mathcal{R} M_{u} f_{k}\right\|_{H_{\mu}^{\infty}}=0$. By (7), we have

$$
f_{k}\left(z_{k}\right)=0, \mathcal{R} f_{k}\left(z_{k}\right)=A \frac{\left|z_{k}\right|^{2}}{\left(1-\left|z_{k}\right|^{2}\right)^{\frac{n+1+q}{p}}}
$$

so

$$
\begin{aligned}
& |A| \frac{\mu\left(\left|z_{k}\right|\right)\left|u\left(z_{k}\right)\right|\left|z_{k}\right|^{2}}{\left(1-\left|z_{k}\right|^{2}\right)^{\frac{n+1+q}{p}}} \\
& =\mu\left(\left|z_{k}\right|\right)\left|\mathcal{R} f_{k}\left(z_{k}\right) u\left(z_{k}\right)+f_{k}\left(z_{k}\right) \mathcal{R} u\left(z_{k}\right)\right| \\
& \leq\left\|\mathcal{R} M_{u} f_{k}\right\|_{H_{\mu}^{\infty}} \rightarrow 0, \text { as } k \rightarrow \infty .
\end{aligned}
$$

Hence

$$
\lim _{k \rightarrow \infty} \frac{\mu\left(\left|z_{k}\right|\right)\left|u\left(z_{k}\right)\right|}{\left(1-\left|z_{k}\right|^{2}\right)^{\frac{n+1+q}{p}}}=\lim _{k \rightarrow \infty} \frac{\mu\left(\left|z_{k}\right|\right)\left|u\left(z_{k}\right)\right|\left|z_{k}\right|^{2}}{\left(1-\left|z_{k}\right|^{2}\right)^{\frac{n+1+q}{p}}}=0,
$$

which means that (15) holds. To prove (14), we set $g_{k}(z)=g_{z_{k}}(z)$, that is

$$
g_{k}(z)=\frac{1-\left|z_{k}\right|^{2}}{\left(1-\left\langle z, z_{k}\right\rangle\right)^{\frac{n+1+q}{p}}}, z \in \mathbb{B} .
$$

It is obvious $g_{k} \in F(p, q, s), \sup _{k \in \mathbb{N}}\left\|g_{k}\right\|_{F(p, q, s)} \leq C$ and $g_{k}$ converges to zero uniformly on the compact subsets of $\mathbb{B}$. By Lemma 4 , we have $\lim _{k \rightarrow \infty}\left\|\mathcal{R} M_{u} g_{k}\right\|_{H_{\mu}^{\infty}}=0$.

By (12), we have

$$
g_{k}\left(z_{k}\right)=\frac{1}{\left(1-\left|z_{k}\right|^{2}\right)^{\frac{n+1+q}{p}}-1}, \mathcal{R} g_{k}\left(z_{k}\right)=A \frac{\left|z_{k}\right|^{2}}{\left(1-\left|z_{k}\right|^{2}\right)^{\frac{n+1+q}{p}}},
$$


so

$$
\begin{aligned}
& \frac{\mu\left(\left|z_{k}\right|\right)\left|\mathcal{R} u\left(z_{k}\right)\right|}{\left(1-\left|z_{k}\right|^{2}\right)^{\frac{n+1+q}{p}-1}} \\
\leq & \left\|\mathcal{R} M_{u}\left(g_{k}\right)\right\|_{H_{\mu}^{\infty}}+|A| \frac{\mu\left(\left|z_{k}\right|\right)\left|u\left(z_{k}\right)\right|\left|z_{k}\right|^{2}}{\left(1-\left|z_{k}\right|^{2}\right)^{\frac{n+1+q}{p}}} \\
& \rightarrow 0, \text { as } k \rightarrow \infty,
\end{aligned}
$$

hence (14) holds.

$(C) \Rightarrow(B)$ Assume that (14) and (15) hold. Then by using (3), for every $f \in$ $F(p, q, s)$, we have

$$
\mu(|z|)\left|\mathcal{R} M_{u} f(z)\right| \rightarrow 0, \text { as }|z| \rightarrow 1 .
$$

Hence $\mathcal{R} M_{u} f \in H_{\mu, 0}^{\infty}$. By Theorem 1, the operator $\mathcal{R} M_{u}: F(p, q, s) \rightarrow H_{\mu}^{\infty}$ is bounded, so that the operator $\mathcal{R} M_{u}: F(p, q, s) \rightarrow H_{\mu, 0}^{\infty}$ is bounded. And for every $\varepsilon>0$, there is a $\delta \in(0,1)$, such that

$$
\frac{\mu(|z|)|\mathcal{R} u(z)|}{\left(1-|z|^{2}\right)^{\frac{q+n+1}{p}-1}}<\varepsilon
$$

and

$$
\frac{\mu(|z|)|u(z)|}{\left(1-|z|^{2}\right)^{\frac{n+1+q}{p}}}<\varepsilon,
$$

for $\delta<|z|<1$. Let $\left\{a_{k}\right\} \subset F(p, q, s), \sup _{k \in \mathbb{N}}\left\|a_{k}\right\|_{F(p, q, s)} \leq C$ and $a_{k}$ converge to zero uniformly on the compact subsets of $\mathbb{B}$, by the Cauchy integral estimates, we have that $\mathcal{R} a_{k}$ also converges to zero uniformly on the compact subsets of $\mathbb{B}$. Hence, we have

$$
\begin{aligned}
& \left\|\left(\mathcal{R} M_{u}\right) a_{k}\right\|_{H_{\mu}^{\infty}}=\sup _{z \in \mathbb{B}} \mu(|z|)\left|\left(\mathcal{R} M_{u} a_{k}\right)(z)\right| \\
= & \sup _{z \in \mathbb{B}} \mu(|z|)\left|\mathcal{R} u(z) a_{k}(z)+u(z) \mathcal{R} a_{k}(z)\right| \\
\leq & \left(\sup _{|z| \leq \delta}+\sup _{\delta<|z|<1}\right) \mu(|z|)\left|\mathcal{R} u(z) a_{k}(z)+u(z) \mathcal{R} a_{k}(z)\right| \\
\leq & \sup _{|z| \leq \delta} \mu(|z|)\left|\mathcal{R} u(z) a_{k}(z)+u(z) \mathcal{R} a_{k}(z)\right| \\
& +\sup _{\delta<|z|<1}\left(\frac{\mu(|z|)|\mathcal{R} u(z)|}{\left(1-|z|^{2}\right)^{\frac{q+n+1}{p}-1}}+\frac{\mu(|z|)|u(z)|}{\left(1-|z|^{2}\right)^{\frac{n+1+q}{p}}}\right)\left\|a_{k}\right\|_{F(p, q, s)} .
\end{aligned}
$$

By (20)-(22) and since the sequences $a_{k}(z)$ and $\mathcal{R} a_{k}(z)$ converge to zero uniformly on the compact set $\{z \in \mathbb{B}:|z| \leq \delta\}$, we have that for sufficiently large $k$

$$
\left\|\left(\mathcal{R} M_{u}\right) a_{k}\right\|_{H_{\mu}^{\infty}} \leq \varepsilon+C \varepsilon .
$$


Applying Lemma 4, we can get the operator $\mathcal{R} M_{u}: F(p, q, s) \rightarrow H_{\mu, 0}^{\infty}$ is compact.

Case 3.2. $p=q+n+1$

Theorem 3. Assume that $0<p, s<\infty,-n-1<q<\infty, q+s>-1, p=q+n+1$ and $\mu$ is a normal weight, then, $\mathcal{R} M_{u}: F(p, q, s) \rightarrow H_{\mu}^{\infty}$ is bounded if and only if

$$
\sup _{z \in \mathbb{B}} \frac{\mu(|z|)|u(z)|}{1-|z|^{2}}<\infty
$$

and

$$
\sup _{z \in \mathbb{B}} \mu(|z|)|\mathcal{R} u(z)| \cdot \log \frac{2}{1-|z|^{2}}<\infty
$$

Proof. First we assume that conditions (23) and (24) hold. For any $f \in F(p, q, s)$, by Lemma 1 and Lemma 2, we have

$$
\begin{aligned}
& \mu(|z|)\left|\mathcal{R} M_{u} f(z)\right| \\
= & \mu(|z|)|u(z) \mathcal{R} f(z)+\mathcal{R} u(z) f(z)| \\
\leq & C\|f\|_{\mathcal{B}}\left(\frac{\mu(|z|)|u(z)|}{1-|z|^{2}}+\mu(|z|)|\mathcal{R} u(z)| \cdot \log \frac{2}{1-|z|^{2}}\right) \\
\leq & C\|f\|_{F(p, q, s)}\left(\frac{\mu(|z|)|u(z)|}{1-|z|^{2}}+\mu(|z|)|\mathcal{R} u(z)| \cdot \log \frac{2}{1-|z|^{2}}\right),
\end{aligned}
$$

so that the operator $\mathcal{R} M_{u}: F(p, q, s) \rightarrow H_{\mu}^{\infty}$ is bounded. Conversely, assume that the operator $\mathcal{R} M_{u}: F(p, q, s) \rightarrow H_{\mu}^{\infty}$ is bounded. Then for any $f \in F(p, q, s)$, there is a positive constant $C$ independent of $f$ such that $\left\|\mathcal{R} M_{u} f\right\| \leq C\|f\|_{F(p, q, s)}$. Given any $\omega \in \mathbb{B}$, set

$$
h_{\omega}(z)=\log \frac{2}{1-\langle z \bar{\omega}\rangle}-\frac{\left(\log \frac{2}{1-\langle z \bar{\omega}\rangle}\right)^{2}}{\log \frac{2}{1-|\omega|^{2}}}, z \in \mathbb{B}
$$

then

$$
\mathcal{R} h_{\omega}(z)=\frac{z \bar{\omega}}{1-z \bar{\omega}}-\frac{\left(2 \log \frac{2}{1-z \bar{\omega}}\right)(z \bar{\omega})}{\left(\log \frac{2}{1-|\omega|^{2}}\right)(1-z \bar{\omega})} .
$$

It is known that $h_{\omega}(z) \in F(p, q, s)$ and $\sup _{\omega \in \mathbb{B}}\left\|h_{\omega}\right\|_{F(p, q, s)} \leq C<\infty$ (see [31, 43]), and moreover we have that

$$
h_{\omega}(\omega)=0, \mathcal{R} h_{\omega}(\omega)=-\frac{|\omega|^{2}}{1-|\omega|^{2}}
$$


Hence

$$
\begin{aligned}
& \frac{\mu(|\omega|)|u(\omega)||\omega|^{2}}{\left(1-|\omega|^{2}\right)^{\frac{n+1+q}{p}}}=\mu(|\omega|)\left|\mathcal{R} h_{\omega}(\omega) u(\omega)\right| \\
\leq & \mu(|\omega|)\left|\mathcal{R} h_{\omega}(\omega) u(\omega)+\mathcal{R} u(\omega) h_{\omega}(\omega)\right| \\
\leq & \left\|\mathcal{R} M_{u} h_{\omega}\right\|_{H_{\mu}^{\infty}} \leq C\left\|\mathcal{R} M_{u}\right\|_{F(p, q, s) \rightarrow H_{\mu}^{\infty}} .
\end{aligned}
$$

Similar to the proof of (2) in Theorem 1, (23) holds. To prove (24), we set

$$
l_{\omega}(z)=\log \frac{2}{1-\langle z \bar{\omega}\rangle}, \quad z \in \mathbb{B},
$$

then

$$
\mathcal{R} l_{\omega}(z)=\frac{z \bar{\omega}}{1-z \bar{\omega}} .
$$

It is known that $l_{\omega}(z) \in F(p, q, s)$, by Lemma 5 , we can see $\sup _{\omega \in \mathbb{B}}\left\|l_{\omega}\right\|_{F(p, q, s)} \leq C$, and we have

$$
l_{\omega}(\omega)=\log \frac{2}{1-|\omega|^{2}}, \mathcal{R} l_{\omega}(\omega)=\frac{|\omega|^{2}}{1-|\omega|^{2}}
$$

so

$$
\begin{aligned}
& \mu(|\omega|)|\mathcal{R} u(\omega)| \cdot \log \frac{2}{1-|\omega|^{2}} \\
\leq & \mu(|\omega|)\left|\mathcal{R} u(\omega) l_{\omega}(\omega)+\mathcal{R} l_{\omega}(\omega) u(\omega)\right|+\frac{\mu(|\omega|)|u(\omega)| \cdot|\omega|^{2}}{1-|\omega|^{2}} \\
\leq & \left\|\mathcal{R} M_{u} l_{\omega}\right\|_{H_{\mu}^{\infty}}+C \\
\leq & \left\|\mathcal{R} M_{u}\right\|_{F(p, q, s) \rightarrow H_{\mu}^{\infty}}+C,
\end{aligned}
$$

which means that (24) holds.

Theorem 4. Assume that $0<p, s<\infty,-n-1<q<\infty, q+s>-1, p=q+n+1$ and $\mu$ is a normal weight, then the following statements are equivalent:

$(A) \mathcal{R} M_{u}: F(p, q, s) \rightarrow H_{\mu}^{\infty}$ is compact;

$(B) \mathcal{R} M_{u}: F(p, q, s) \rightarrow H_{\mu, 0}^{\infty}$ is compact;

$(C)$

$$
\lim _{|z| \rightarrow 1} \frac{\mu(|z|)|u(z)|}{1-|z|^{2}}=0
$$

and

$$
\lim _{|z| \rightarrow 1} \mu(|z|)|\mathcal{R} u(z)| \cdot \log \frac{2}{1-|z|^{2}}=0
$$


Proof. $(B) \Rightarrow(A)$. This implication is obvious.

$(A) \Rightarrow(C)$ Suppose that the operator $\mathcal{R} M_{u}: F(p, q, s) \rightarrow H_{\mu}^{\infty}$ is compact, then $\mathcal{R} M_{u}: F(p, q, s) \rightarrow H_{\mu}^{\infty}$ is bounded. Let $\left\{z_{k}\right\}$ be a sequence in $\mathbb{B}$ such that $\left|z_{k}\right| \rightarrow 1$ as $k \rightarrow \infty$. Set $h_{k}(z)=h_{z_{k}}(z)$, that is

$$
h_{k}(z)=\frac{\left(\log \frac{2}{1-\left\langle z, z_{k}\right\rangle}\right)^{2}}{\log \frac{2}{1-\left|z_{k}\right|^{2}}}-\frac{\left(\log \frac{2}{1-\left\langle z, z_{k}\right\rangle}\right)^{3}}{\left(\log \frac{2}{1-\left|z_{k}\right|^{2}}\right)^{2}}, k \in \mathbb{N} .
$$

Then, $h_{k} \in F(p, q, s), \sup _{k \in \mathbb{N}}\left\|h_{k}\right\|_{F(p, q, s)} \leq C$ and $h_{k}$ converges to zero uniformly on the compact subsets of $\mathbb{B}$ as $k \rightarrow \infty$. By Lemma 4, we have $\lim _{k \rightarrow \infty}\left\|\mathcal{R} M_{u} h_{k}\right\|_{H_{\mu}^{\infty}}=0$, and we can get

So

$$
h_{k}\left(z_{k}\right)=0, \mathcal{R} h_{k}\left(z_{k}\right)=-\frac{\left|z_{k}\right|^{2}}{1-\left|z_{k}\right|^{2}} .
$$

$$
\begin{aligned}
& \frac{\mu\left(\left|z_{k}\right|\right)\left|u\left(z_{k}\right)\right|\left|z_{k}\right|^{2}}{1-\left|z_{k}\right|^{2}} \\
\leq & \mu\left(\left|z_{k}\right|\right)\left|\mathcal{R} h_{k}\left(z_{k}\right) u\left(z_{k}\right)+h_{k}\left(z_{k}\right) \mathcal{R} u\left(z_{k}\right)\right| \\
\leq & \left\|\mathcal{R} M_{u} h_{k}\right\|_{H_{\mu}^{\infty}} \rightarrow 0, \text { as } k \rightarrow \infty,
\end{aligned}
$$

hence, (30) holds. To prove (31), we set

$$
l_{k}(z)=\frac{\left(\log \frac{2}{1-\left\langle z, z_{k}\right\rangle}\right)^{2}}{\log \frac{2}{1-\left|z_{k}\right|^{2}}}, k \in \mathbb{N} .
$$

Then, $l_{k} \in F(p, q, s)$, $\sup _{k \in \mathbb{N}}\left\|l_{k}\right\|_{F(p, q, s)} \leq C$ and $l_{k}$ converges to zero uniformly on the compact subsets of $\mathbb{B}$ as $k \rightarrow \infty$. By Lemma 4 , we have $\lim _{k \rightarrow \infty}\left\|\left(\mathcal{R} M_{u}\right) l_{k}\right\|_{H_{\mu}^{\infty}}=0$, and moreover we have that

$$
l_{k}\left(z_{k}\right)=\log \frac{2}{1-\left|z_{k}\right|^{2}}, \mathcal{R} l_{k}\left(z_{k}\right)=2 \frac{\left|z_{k}\right|^{2}}{1-\left|z_{k}\right|^{2}},
$$

so

$$
\begin{aligned}
& \mu\left(\left|z_{k}\right|\right)\left|\mathcal{R} u\left(z_{k}\right)\right| \cdot \log \frac{2}{1-\left|z_{k}\right|^{2}} \\
\leq & \mu\left(\left|z_{k}\right|\right)\left|\mathcal{R} l_{k}\left(z_{k}\right) u\left(z_{k}\right)+l_{k}\left(z_{k}\right) \mathcal{R} u\left(z_{k}\right)\right|+2 \frac{\mu\left(\left|z_{k}\right|\right)\left|u\left(z_{k}\right)\right|\left|z_{k}\right|^{2}}{1-\left|z_{k}\right|^{2}} \\
\leq & \left\|\mathcal{R} M_{u}\left(l_{k}\right)\right\|_{H_{\mu}^{\infty}}+2 \frac{\mu\left(\left|z_{k}\right|\right)\left|u\left(z_{k}\right)\right|\left|z_{k}\right|^{2}}{1-\left|z_{k}\right|^{2}} \\
& \rightarrow 0, \text { as } k \rightarrow \infty
\end{aligned}
$$


which means that (31) holds.

$(C) \Rightarrow(B)$ Assume that (30) and (31) hold. Similar to Theorem 2, the operator $\mathcal{R} M_{u}: F(p, q, s) \rightarrow H_{\mu, 0}^{\infty}$ is bounded. And for every $\varepsilon>0$, there is a $\delta \in(0,1)$, such that

$$
\mu(|z|)|\mathcal{R} u(z)| \cdot \log \frac{2}{1-|z|^{2}}<\varepsilon,
$$

and

$$
\frac{\mu(|z|)|u(z)|}{1-|z|^{2}}<\varepsilon
$$

whenever $\delta<|z|<1$. Let $b_{k} \subset F(p, q, s), \sup _{k \in \mathbb{N}}\left\|b_{k}\right\|_{F(p, q, s)} \leq C$ and $b_{k}$ converge to zero uniformly on the compact subsets of $\mathbb{B}$, by the Cauchy integral estimates, we have that $\mathcal{R} b_{k}$ also converges to zero uniformly on the compact subsets of $\mathbb{B}$. Hence,

$$
\begin{aligned}
& \left\|\left(\mathcal{R} M_{u}\right) b_{k}\right\|_{H_{\mu}^{\infty}}=\sup _{z \in \mathbb{B}} \mu(|z|)\left|\left(\mathcal{R} M_{u}\right) b_{k}(z)\right| \\
\leq & \sup _{z \in \mathbb{B}} \mu(|z|)\left|\mathcal{R} u(z) b_{k}(z)+u(z) \mathcal{R} b_{k}(z)\right| \\
\leq & \sup _{|z| \leq \delta} \mu(|z|)\left|\mathcal{R} u(z) b_{k}(z)+u(z) \mathcal{R} b_{k}(z)\right| \\
& \quad+\sup _{\delta<|z|<1} \mu(|z|)\left|\mathcal{R} u(z) b_{k}(z)+u(z) \mathcal{R} b_{k}(z)\right| \\
\leq & \sup _{|z| \leq \delta} \mu(|z|)\left|\mathcal{R} u(z) b_{k}(z)+u(z) \mathcal{R} b_{k}(z)\right| \\
& +C \sup _{\delta<|z|<1}\left(\mu(|z|)|\mathcal{R} u(z)| \cdot \log \frac{2}{1-|z|^{2}}+\frac{\mu(|z|)|u(z)|}{1-|z|^{2}}\right) .
\end{aligned}
$$

By (36)-(38), since the sequences $b_{k}(z)$ and $\mathcal{R} b_{k}(z)$ converge to zero uniformly on the compact set $\{z \in \mathbb{B}:|z| \leq \delta\}$, we have

$$
\lim _{k \rightarrow \infty}\left\|\left(\mathcal{R} M_{u}\right) b_{k}\right\|_{H_{\mu}^{\infty}}=0 .
$$

Applying Lemma 4, we get $\mathcal{R} M_{u}: F(p, q, s) \rightarrow H_{\mu, 0}^{\infty}$ is compact.

Case 3.3. $p>q+n+1$

Theorem 5. Assume that $0<p, s<\infty,-n-1<q<\infty, q+s>-1, p>q+n+1$ and $\mu$ is a normal weight, then $\mathcal{R} M_{u}: F(p, q, s) \rightarrow H_{\mu}^{\infty}$ is bounded if and only if $u \in \mathcal{B}_{\mu}$ and

$$
\sup _{z \in \mathbb{B}} \frac{\mu(|z|)|u(z)|}{\left(1-|z|^{2}\right)^{\frac{n+1+q}{p}}}<\infty .
$$


Proof. First let us assume that conditions $u \in \mathcal{B}_{\mu}$ and (39) hold. For any $f \in$ $F(p, q, s)$, by Lemma 1 and Lemma 2, we have

$$
\begin{aligned}
& \mu(|z|)\left|\left(\mathcal{R} M_{u} f\right)(z)\right| \\
= & \mu(|z|)|\mathcal{R} u(z) f(z)+u(z) \mathcal{R} f(z)| \\
\leq & C\|f\|_{\mathcal{B}^{\frac{n+1+q}{p}}}\left(\mu(|z|)|\mathcal{R} u(z)|+\frac{\mu(|z|)|u(z)|}{\left(1-|z|^{2}\right)^{\frac{n+1+q}{p}}}\right) \\
\leq & C\|f\|_{F(p, q, s)}\left(\mu(|z|)|\mathcal{R} u(z)|+\frac{\mu(|z|)|u(z)|}{\left(1-|z|^{2}\right)^{\frac{n+1+q}{p}}}\right) .
\end{aligned}
$$

It follows that the operator $\mathcal{R} M_{u}: F(p, q, s) \rightarrow H_{\mu}^{\infty}$ is bounded. Conversely, suppose the operator $\mathcal{R} M_{u}: F(p, q, s) \rightarrow H_{\mu}^{\infty}$ is bounded. Then for any $f \in F(p, q, s)$, there is a positive constant $C$ independent of $f$ such that $\left\|\left(\mathcal{R} M_{u}\right) f\right\|_{H_{\mu}^{\infty}} \leq C\|f\|_{F(p, q, s)}$. For $f \equiv 1$, we have that $u \in \mathcal{B}_{\mu}$. Similar to the proof of (2), (39) follows.

Theorem 6. Assume that $0<p, s<\infty,-n-1<q<\infty, q+s>-1$, $p>q+n+1, \mu$ is a normal weight, then the following statements are equivalent:

(A) $\mathcal{R} M_{u}: F(p, q, s) \rightarrow H_{\mu}^{\infty}$ is compact;

(B) $u \in \mathcal{B}_{\mu}$ and

$$
\lim _{|z| \rightarrow 1} \frac{\mu(z)|u(z)|}{\left(1-|z|^{2}\right)^{\frac{n+1+q}{p}}}=0 .
$$

Proof. $(A) \Rightarrow(B)$. We assume that $\mathcal{R} M_{u}: F(p, q, s) \rightarrow H_{\mu}^{\infty}$ is compact. Then for $f \equiv 1$, we obtain that $u \in \mathcal{B}_{\mu}$. Exploiting the test function in (16), similarly to the proof of Theorem 2, we obtain (41) holds.

$(B) \Rightarrow(A)$. Assume that $\left\{c_{k}\right\}_{k \in \mathbb{N}}$ is a sequence in $F(p, q, s)$ such that $\sup _{k \in \mathbb{N}}\left\|c_{k}\right\|_{F(p, q, s)} \leq$ $C$, and $c_{k} \rightarrow 0$ uniformly on the compact subsets of $\mathbb{B}$ as $k \rightarrow \infty$. By (41), we have for any $\varepsilon>0$, there is a $\delta \in(0,1)$, when $\delta<|z|<1$,

$$
\frac{\mu(|z|)|u(z)|}{\left(1-|z|^{2}\right)^{\frac{n+1+q}{p}}}<\varepsilon .
$$

From (42) we have that for sufficiently large $k$

$$
\begin{aligned}
& \left\|\left(\mathcal{R} M_{u}\right) c_{k}\right\|_{H_{\mu}^{\infty}}=\sup _{z \in \mathbb{B}} \mu(|z|)\left|\left(\mathcal{R} M_{u} c_{k}\right)(z)\right| \\
= & \sup _{z \in \mathbb{B}} \mu(|z|)\left|\mathcal{R} c_{k}(z) u(z)+\mathcal{R} u(z) c_{k}(z)\right| \\
\leq & \sup _{|z| \leq \delta} \mu(|z|)\left|\mathcal{R} c_{k}(z) u(z)+\mathcal{R} u(z) c_{k}(z)\right| \\
& +\sup _{\delta<|z|<1} \mu(|z|)\left|\mathcal{R} c_{k}(z) u(z)\right|+\|u\|_{\mathcal{B}_{\mu}} \sup _{z \in \mathbb{B}}\left|c_{k}(z)\right|
\end{aligned}
$$




$$
\begin{aligned}
& \leq \varepsilon+C \sup _{\delta<|z|<1} \frac{\mu(|z|)|u(z)|}{\left(1-|z|^{2}\right)^{\frac{n+1+q}{p}}}+\|u\|_{\mathcal{B}_{\mu}} \sup _{z \in \mathbb{B}}\left|c_{k}(z)\right| \\
& \leq \varepsilon+C \varepsilon+\|u\|_{\mathcal{B}_{\mu}} \sup _{z \in \mathbb{B}}\left|c_{k}(z)\right| .
\end{aligned}
$$

Since $p>q+n+1$ then

$$
\int_{0}^{1} \frac{d t}{\left(1-t^{2}\right)^{\frac{n+1+q}{p}}}<+\infty .
$$

Applying the corresponding result for the $\mu$-Bloch space (see [36, Lemma 4.2]), we also have

$$
\lim _{k \rightarrow \infty} \sup _{z \in \mathbb{B}}\left|c_{k}(z)\right|=0 .
$$

From (43) it follows that $\lim _{k \rightarrow \infty}\left\|\mathcal{R} M_{u} c_{k}\right\|_{H_{\mu}^{\infty}}=0$, so that the operator $\mathcal{R} M_{u}: F(p, q, s)$ $\rightarrow H_{\mu}^{\infty}$ is compact, finishing the proof of the theorem.

Theorem 7. Assume that $0<p, s<\infty,-n-1<q<\infty, q+s>-1$, $p>q+n+1$ and $\mu$ is a normal weight, then the following statements are equivalent:

(A) $\mathcal{R} M_{u}: F(p, q, s) \rightarrow H_{\mu, 0}^{\infty}$ is compact;

(B) $u \in \mathcal{B}_{\mu, 0}$ and

$$
\lim _{|z| \rightarrow 1} \frac{\mu(|z|)|u(z)|}{\left(1-|z|^{2}\right)^{\frac{n+1+q}{p}}}=0 .
$$

Proof. $(A) \Rightarrow(B)$. We assume that $\mathcal{R} M_{u}: F(p, q, s) \rightarrow H_{\mu, 0}^{\infty}$ is compact. For $f \equiv 1$, we obtain that $u \in \mathcal{B}_{\mu, 0}$. In the same way as in Theorem 6 , we obtain that (44) holds.

$(B) \Rightarrow(A)$. By Lemma 1 and Lemma 2, we have

$$
\begin{aligned}
& \mu(|z|)\left|\left(\mathcal{R} M_{u} f\right)(z)\right|=\mu(|z|)|\mathcal{R} f(z) u(z)+\mathcal{R} u(z) f(z)| \\
& \leq C\|f\|_{F(p, q, s)} \frac{\mu(|z|)|u(z)|}{\left(1-|z|^{2}\right)^{\frac{n+1+q}{p}}}+C\|f\|_{F(p, q, s)} \mu(|z|)|\mathcal{R} u(z)| .
\end{aligned}
$$

This along with Theorem 5 implies that $\mathcal{R} M_{u}\left\{f:\|f\|_{F(p, q, s)} \leq 1\right\}$ is bounded. Taking the supremum over the unit ball in $F(p, q, s)$. Letting $|z| \rightarrow 1$ in (45), using the condition $(B)$, and by applying Lemma 3 , we get the compactness of the operator $\mathcal{R} M_{u}: F(p, q, s) \rightarrow H_{\mu, 0}^{\infty}$. This completes the proof of the theorem.

\section{ACKNOWLEDGMENTS}

The authors wish to thank the referee(s) for carefully reading the manuscript and making several useful suggestions for improvement, and for bringing to our attention important references. 


\section{REFERENCES}

1. K. L. Avetisyan, Hardy-Bloch type spaces and lacunary series on the polydisk, Glas. Math. J., 49(2) (2007), 345-356.

2. K. D. Bierstedt and W. H. Summers, Biduals of weighted Banach spaces of anzlytic functions, J. Austr. Mathe. Soc., 54(1) (1993), 70-79.

3. X. Fu and X. Zhu, Weighted composition operators on some weighted spaces in the unit ball, Abstr. Appl. Anal., 2006 (2006), Article ID 605807, 8 pp.

4. Z. Hu, Extended Cesàro operators on mixed norm spaces, Proc. Amer. Math. Soc., 131(7) (2003), 2171-2179, (electronic).

5. B. Li and C. Ouyang, Higher radial derivative of functions of $Q_{p}$ spaces and its applications, J. Math. Anal. Appl., 327(2) (2007), 1257-1272.

6. S. Li, Riemann-Stieltjes operators from $F(p, q, s)$ spaces to $\alpha$-Bloch spaces on the unit ball, J. Inequal. Appl., 2006 (2006), Article ID 27874, 14 pp.

7. S. Li and S. Stevic, Composition followed by differentiation between Bloch type spaces, J. Comput. Anal. Appl., 9(2) (2007), 195-206.

8. S. Li and S. Stevic, Composition followed by differentiation from mixed-norm spaces to $\alpha$-Bloch spaces, Sb. Math., 199(11/12) (2008), 1847-1857.

9. S. Li and S. Stevic, Generalized composition operators on Zygmund spaces and Bloch type spaces, J. Math. Anal. Appl., 338(2) (2008), 1282-1295.

10. S. Li and S. Stević, Compactness of Riemann-Stieltjes operators between $F(p, q, s)$ and $\alpha$-Bloch spaces, Publ. Math. Debrecen, 72(1/2) (2008), 111-128.

11. S. Li and S. Stevié, Composition followed by differentiation between $H^{\infty}$ and $\alpha$-Bloch spaces (English summary), Houston J. Math., 35(1) (2009), 327-340.

12. X. Liu and Y. Yu, The product of differentiation operator and multiplication operator from $H^{\infty}$ to Zygmund spaces, J. Xuzhou Norm. Univ. Nat. Sci. Ed., 29(1) (2011), 37-39.

13. Y. Liu, Boundedness of the Bergman type operators on mixed norm spaces, Proc. Amer. Math. Soc., 130(8) (2002), 2363-2367 (electronic).

14. Y. Liu and Y. Yu, Weighted differentiation composition operators from mixed-norm to Zygmund spaces, Numer. Funct. Anal. Optim., 31(8) (2010), 936-954.

15. Y. Liu and Y. Yu, On compactness for iterated commutators, Acta Math. Sci. Ser. B Engl. Ed., 31B(2) (2011), 491-500.

16. Y. Liu and Y. Yu, Composition followed by differentiation between $H^{\infty}$ and Zygmund spaces, Complex. Anal. Oper. Theory, 6(1) (2012), 121-137.

17. Y. Liu and J. Zhou, On an operator $M_{u} \mathcal{R}$ from mixed norm spaces to Zygmund-type spaces on the unit ball, Complex. Anal. Oper. Theory, DOI 10.1007/s11785-012-0237-7. 
18. X. Lv and X. Tang, Extended Cesàro operators from $F(p, q, s)$ spaces to Bloch-type spaces in the unit ball, Commun. Korean Math. Soc., 24(1) (2009), 57-66.

19. K. Madigan and A. Matheson, Compact composition operators on the Bloch space, Trans. Amer. Math. Soc., 347(7) (1995), 2679-2687.

20. W. Rudin, Function Theory in the Unit Ball of $\mathbb{C}^{n}$, Springer-Verlag, New York-Berlin, 1980.

21. A. L. Shields and D. L. Williams, Bounded projections, duality, and multipliers in spaces of analytic functions, Trans. Amer. Math. Soc., 162 (1971), 287-302.

22. S. Stević, On an integral operator on the unit ball in $\mathbb{C}^{n}$, J. Inequal. Appl., 2005(1) (2005), 81-88.

23. S. Stević, Boundedness and compactness of an integral operator in a mixed norm space on the polydisc, Siberian Math. J., 48(3) (2007), 559-569.

24. S. Stevic, On a new integral-type operator from the Bloch space to Bloch-type spaces on the unit ball, J. Math. Anal. Appl., 354(2) (2009), 426-434.

25. S. Stevic, Weighted differentiation composition operators from mixed-norm spaces to weighted-type spaces, Appl. Math. Comput., 211(1) (2009), 222-233.

26. S. Stević, Products of composition and differentiation operators on the weighted Bergman space, Bull. Belg. Math. Soc. Simon Stevin, 16(4) (2009), 623-635.

27. S. Stević, Weighted iterated radial composition operators between some spaces of holomorphic functions on the unit ball, Abstr. Appl. Anal., 2010 (2010), Article ID 801264, $14 \mathrm{pp}$.

28. S. Stević, Composition followed by differentiation from $H^{\infty}$ and the Bloch space to $n$th weighted-type spaces on the unit disk, Appl. Math. Comput., 216(12) (2010), 3450-3458.

29. S. Stević, Weighted differentiation composition operators from $H^{\infty}$ and Bloch spaces to $n$th weigthed-type spaces on the unit disk, Appl. Math. Comput., 216(12) (2010), 3634-3641.

30. S. Stević, On a product-type operator from Bloch spaces to weighted-type spaces on the unit ball, Appl. Math. Comput., 217(12) (2011), 5930-5935.

31. S. Stević, On some integral-type operators between a general space and Bloch-type spaces, Appl. Math. Comput., 218(6) (2011), 2600-2618.

32. S. Stevic, Characterizations of composition followed by differentiation between Blochtype spaces, Appl. Math. Comput., 218(8) (2011), 4312-4316.

33. S. Stević, Boundedness and compactness of an integral-type operator from Bloch-type spaces with normal weights to $F(p, q, s)$ space, Appl. Math. Comput., 218(9) (2012), 5414-5421.

34. S. Stevic, Weighted radial operator from the mixed-norm space to the $n$th weighted-type space on the unit ball, Appl. Math. Comput., 218(18) (2012), 9241-9247. 
35. S. Stević and A. K. Sharma, Iterated differentiation followed by composition from Blochtype spaces to weighted BMOA spaces, Appl. Math. Comput., 218(7) (2011), 35743580 .

36. X. Tang, Extended Cesàro operators between Bloch-type spaces in the unit ball of $\mathbb{C}^{n}$, J. Math. Anal. Appl., 326(2) (2007), 1199-1211.

37. W. Yang, Products of composition and differentiation operators from $Q_{K}(p, q)$ spaces to Bloch-type spaces, Abstr. Appl. Anal., 2009 (2009), Article ID 741920, 14 pp.

38. W. Yang, Composition operators from $F(p, q, s)$ spaces to the $n$th weighted-type spaces on the unit disc, Appl. Math. Comput., 218(4) (2011), 1443-1448.

39. W. Yang, Generalized weighted composition operatora from $F(p, q, s)$ to the Bloch-type space, Appl. Math. Comput., 218(9) (2012), 4967-4972.

40. W. Yang and X. Meng, Generalized composition operators from $F(p, q, s)$ space to Bloch-type spaces, Appl. Math. Comput., 217 (2010), 2513-2519.

41. S. Ye, Weighted composition operator from the general function space $F(p, q, s)$ into logarithmic Bloch spaces, J. Korean Math. Soc., 45(4) (2008), 977-991.

42. Y. Yu, Volterra-type composition operators from logarithmic Bloch spaces into Blochtype spaces, J. Xuzhou Norm. Univ. Nat. Sci. Ed., 27(3) (2009), 14-18.

43. X. Zhang, Multipliers on some holomorphic function spaces, Chinese Ann. Math. Ser. $A$, 26(4) (2005), 477-486.

44. X. Zhang and J. Xiao, Weighted composition operator between two analytic function spaces, Chinese Adv. Math., 35(4) (2006), 477-486.

45. X. Zhang, J. Xiao and Z. Hu, The multipliers between the mixed norm spaces in $\mathbb{C}^{n}, J$. Math. Anal. Appl., 311(2) (2005), 664-674.

46. R. Zhao, On a general family of function space, Ann. Acad. Sci. Fenn. Math. Dissertationes, 1996.

47. Z. Zhou and R. Chen, Weighted composition operators from $F(p, q, s)$ to Bloch type space, Int. Mod. Math., 3(3) (2008), 327-336.

48. K. Zhu, Spaces of Holomorphic Functions in the Unit Ball, Graduate Text in Mathematics 226, Springer, New York, 2005.

49. X. Zhu, Products of differentiation, composition and multiplication from Bergman type spaces to Bers type spaces (English summary), Integral Transforms Spec. Funct., 18(3/4) (2007), 223-231.

50. X. Zhu, Weighted composition operators from $F(p, q, s)$ spaces to $H_{\mu}^{\infty}$ spaces, Abstr. Appl. Anal., 2009 (2009), Article ID 290978, 14 pp. 
Jie Zhou and Yongmin Liu Department of Mathematics Jiangsu Normal University Xuzhou 221116

P. R. China

E-mail: minliu@jsnu.edu.cn 